\title{
Computer Network Reliability Analysis based on Intelligent Computing
}

\author{
Xingyu Luo \\ Chongqing institute of post and telecommunications institute of released \\ Chongqing,China
}

\begin{abstract}
In recent years, with the changes of the times and the continuous development of the society, the development of information technology is also showing a rapid development. The emergence and development of information technology has greatly changed people's living habits and behavior patterns, in people's daily life, study and work play an irreplaceable role. The modern society is developing in the direction of the network, the new era, with the popularization of computer network, people have more and more high reliability requirements, which is always the research hotspot in computer field. Based on this, this paper makes a deep analysis on the reliability of the computer network based on Intelligent Computing.
\end{abstract}

Keywords-intelligent computing; computer network; reliability

\section{INTRODUCTION}

Intelligent computing is a kind of program based on computer, which has the characteristic of thinking. Intelligent computing and artificial intelligence technology to combine together, making the experience of intelligent computing to improve, so as to better help human analysis and solve practical problems. Twenty-first Century is the information age, information technology in various fields are playing an irreplaceable role. From the present stage, information technology has become an important factor to measure the comprehensive strength of the country, and the special background of the times and promote the rapid development of information technology. In order to meet the needs of human society, the control system is changed from the traditional simplification to the modern complex. In view of this situation, the relevant staff to increase the reliability of the research efforts, and achieved remarkable results. Based on many years of work experience, I have a superficial understanding of the computer network reliability.

\section{INFLUENCING FACTORS OF COMPUTER NETWORK} RELIABILITY

\section{A. Terminal equipment}

The so-called terminal equipment in fact is the user equipment, and users can directly contact. The terminal equipment directly determines whether the computer network is safe and reliable. In general, the ability to interact with the terminal equipment, we can say that the higher the reliability of computer networks. With the continuous updating and development of information technology, the terminal equipment has also made progress. Although the development of the terminal equipment is amazing, but in the terminal equipment, the terminal equipment problems are more, and these problems seriously affect the reliability of computer network. In order to improve the reliability of computer networks, we need to start from the improvement of the terminal equipment, in order to ensure the update speed, to improve the reliability of the terminal equipment, as far as possible to reduce the operation of the problem.

\section{B. Transmission equipment}

Transmission equipment is divided into two parts, one is transmission line, the other is transmission equipment. In the actual operation, if it is because the transmission line problems lead to the computer network failure, is not easy to find. Sometimes in order to find fault, need to spend a lot of human resources, the workload of the staff is also large, both time-consuming and laborious. Therefore, when the transmission equipment is installed, the communication line must be chosen according to the requirement of the standard, choose the reliable and standard wiring system, and need to take the fault tolerance and redundancy capability into account. If the condition allows, the routing method is best to choose a dual line. Such as the failure of the network through the network to switch, it can guarantee the normal operation of the transmission equipment, so as to improve the reliability of computer networks.

\section{Network management}

Daily life of some of the large network equipment we are exposed to, and some of the equipment and products are all made by different manufacturers, and this situation caused the structural complexity of the network system. Information will be lost in the daily operation, in order to prevent the occurrence of such cases, to ensure that information in the transmission process of security, accurate, need to rely on the network management technology real-time acquisition of network parameters, timely troubleshooting.

\section{Extension structure}

The so-called extension structure, simply by using the transmission medium to achieve the connection and layout of multiple devices, is reflected in the physical level. The extension structure plays an important role in the computer network reliability, which plays a role in the whole network. The factors that affect the structure of the extension are many, including the size of the network, the transmission medium and the distribution of the users. In 
recent years, the users of the network performance requirements are increasingly high, therefore, the extension of the structure of the task is also more difficult, to meet the needs of users of various network.

\section{COMPUTER NETWORK RELIABILITY BASED ON}

\section{INTELLIGENT COMPUTING}

The whole computer network system is composed of two parts, one is the node and the other is the arc of the connecting node. The nodes are three kinds of input nodes, output nodes and intermediate nodes. The input nodes are only the output arcs are not present in the input arcs. The output nodes are the only input arcs which are not present in the input. Network also has three kinds of forms, which are directed, undirected, and hybrid network. If the network is composed of a directed arc, it is directed to the network. If the network is composed of non directed arcs, the network is composed of a hybrid network. In the work we often meet the structure of the complex network system, in order to make an accurate analysis of its reliability, usually will choose the network diagram to express. It is necessary to make some simple analysis: the state of the system and arc can only be two kinds of non normal fault; no direction is different, but the reliability is the same; if an arc fault, other arc will not be affected, still can be used normally.

\section{COMPUTER NETWORK RELIABILITY ANALYSIS}

\section{METHOD}

\section{A. Node traversal method for the most path set}

In general, we can find the minimal path set by the following three methods: the first one, the adjacency matrix method, and the matrix method. Is a matrix for a number of times and multiply operations, in the case of network nodes are not more suitable for the use of this method, the hand can be; but if the network nodes more, it is not suitable for using this method. As a result, the node is more than the amount of computation, the computation time is long, and the requirement of the computer capacity is higher. Second kinds, Boolean determinant method. This method is easy to understand and operate, and it can be realized by using the matrix determinant, but if the network nodes are more, it is not recommended to use, because it is more complicated to use. Third, node traversal method. The method has a clear order, and even if the node is more, it can also be found in these three methods, the application of the method is the most widely used. But the node of the calendar needs more judgment conditions, if not seriously considered, it will produce errors. The method is: from node I input to each point of the beginning of the $\mathrm{L}$, the output point to find all of the smallest of the road set can stop; determine the conditions: the current node and the node is repeated; the most path set whether to find and all the way set is found.

\section{B. Intelligent granular computing of the minimal path set}

Grain located on the theory of domain refers to a bunch of points, if you want to do to more differences and close to is not a easy job, and we also hard to the organic combination of its and one function, can effectively solve the problem, and granular computing and most problems in other fields to be able to find the answer by granular computing. In computer network system in the route set operation steps are as follows: first of all we need to turn the traditional algorithm in two dimensional array is expressed as a one-dimensional array, represented by $\mathrm{n}-1$; Mainly because of $n$ nodes set the path of the road than $n$ 1 small long, or equal to $n-1$, that is, using a one-dimensional array, from the input to the output nodes one by one on each node traversal, and get the result, the path set is found, writes the result to the corresponding files, and start looking for the next most path set, find out later, repeat the above operation; So far has been to set all the path to find out, will be released array; Next, when the array into the calculation process and the dynamic involved in this process, if the budget is completed, needs to be immediately released, the aim is through the release save memory, thus improve the operation efficiency; After completion of the above steps, the path is expressed in node set file, so we need to convert them to arc form transformation and the result was preserved; Finally using smart granularity segmentation, analysis of computer network system is reliable.

\section{THE EFFECTIVE WAYS TO REALIZE THE COMPUTER NETWORK RELIABILITY}

\section{A. Strengthen computer network level, system structure design}

There are two main factors that constitute a computer network, one is hardware, the other is software. Hardware facilities we all know that the network device, such as the central processor, input devices, output devices, etc.. The software infrastructure refers to the level of the network and the composition of the system. Facilities are hardware or software facilities through the visible, invisible to judge, if it is tangible, it is hardware facilities; if it is invisible, it belongs to the software facilities. Although hardware facilities are the basis of a computer, the role and status of the software in the computer network can not be ignored. In order to improve the reliability of computer network, we must pay close attention to the design of its level and system structure. Only scientific design can guarantee the reliability of the level and system structure, and then improve the reliability of computer network.

\section{B. To realize fault tolerance}

First, in the design of computer networks, we must follow the principle of redundancy, parallel. In taking effective measures to ensure that the computer network is in a state of security, the terminal equipment, which is the user equipment and the server are connected to the two computer network center. The purpose of this is to reduce the probability of failure, if there is a sudden situation, users need not worry about the reliability of computer networks, through the above settings, can guarantee the normal operation of the computer; second, in the actual work, if a device fails, other equipment should not be affected, maintain normal operation. And to meet this requirement, we need to link the data link, router and wide area network and other equipment, and finally, the 
information technology update speed, to ensure that the computer network is reliable, we must introduce advanced technology, but also to increase the advanced technology development efforts, will be advanced technology applied to the server, can effectively improve its reliability. In addition, when selecting the server, we must take full consideration, choose carefully, to ensure that the server has a good fault tolerance and high reliability, and its combination with advanced technology to achieve satisfactory results.

\section{Redundancy measures}

To ensure the safety of computer network and reliable, and also need to be redundant, that is to effectively use backup device, in a sudden accident, action of emergency backup device can effectively. This requests us to immediately set out to design based on dual network redundancy. In simple terms, redundancy measures on the basis of a computer network, design a standby computer network to match. Speed up the pace of modern city life, put forward higher requirements for the transmission of information, not only to ensure their effectiveness, and ensure that and timeliness. If you want to conform to the requirements, you need one by one, connect all network points in the whole network structure. In this way can ensure the stable operation of the computer network. Whether in a single network or double road network, its reliability can be guaranteed.

\section{CONCLUSIONS}

In the information era, people put forward the reliability of the computer network is more and more high demand, therefore, to study the problem has very important practical significance, but the research on this issue need further in-depth. Nowadays, the living standard of people more and more high, the computer got popularization, and the computer has become essential to modern society of science and technology products. Along with the trend of the development of information technology presents a complicated gradually, and more and more life, work have been moved to the computer, leading to the reliability of the computer network become the people focus on the topic. The thesis carried on the thorough analysis of the reliability of the computer network, and puts forward some personal advice to improve the system reliability, has a certain reference value.

\section{REFERENCES}

[1] bass. Based on the intelligent calculation of computer network reliability analysis $[\mathrm{J}]$. Journal of wireless technology, 2015 (1).

[2] xiao-bo ren. Based on the intelligent computation of the computer network reliability analysis [J]. Computer CD software and applications, 2014 (18).

[3] Meng Xin. Reliability theory of computer communication network analysis and the multi-objective optimization [J]. Journal of information and computer, 2015 (4).

[4] chui xiaolong. Reliability analysis of computer communication network based on genetic algorithm and optimization [J]. Journal of consumer Tribune, 2015 (5).

[5] Sue. Improve the reliability of computer network operation analysis [J]. Journal of information and communication, 2015 (04).

[6] money really kun. Jiang Zhimeng. Zhou Siji. Computer network reliability analysis and design research [J]. Journal of digital users, 2013(27). 